\section{„3D-Therapie“ bei eosinophiler Ösophagitis}

Ein Krankheitsbild, das zunehmend "prominenter" und auch bei Kindern immer häufiger beobachtet wird, sei die eosinophile Ösophagitis, berichtete PD Dr. Petr Hruz von der Abteilung für Gastroenterologie und Hepatologie des Universitätsspitals Basel. Bei mehr als der Hälfte der Patienten ist eine atopische Diathese assoziiert. Die chronisch-entzündliche, immunvermittelte Erkrankung der Speiseröhre weist histologisch eine dichte Infiltration der Ösophagusschleimhaut mit Eosinophilen auf.

Als Leitsymptome gelten Dysphagie für feste Speisen, Bolusimpaktation und Retrosternalschmerzen. Während Erwachsene häufig Coping-Strategien entwickeln, kommt es bei Kindern oft zu sehr belastenden unspezifischen Folgeproblemen wie Nahrungsverweigerung und Gedeihstörungen, Speiseregurgita- tionen oder Bauchschmerzen. Um die Lebensqualität zu verbessern, aber auch um Spätfolgen wie Fibrosierung und Remodeling des Ösophagusepithels vorzubeugen, sollte eine eosinophile Ösophagitis frühzeitig behandelt werden.

Hruz empfahl eine 3D-Therapiestrategie aus Diät, Drugs und Dilatation: Bei Nahrungsmittelallergien wirkt sich eine entsprechend angepasste Ernährung auch günstig auf Schluckbeschwerden und eine eosinophile Entzündung aus. Eine „Six-Food-Eliminationsdiät“ ohne Kuhmilch, Soja, Eier, Weizen, Nüsse und Meeresfrüchte wurde in den letzten Jahren bei Kindern und Erwachsenen implementiert, und bei einem großen Teil der Patienten konnte eine Verbesserung der Symptome und eine histologische Remission beobachtet werden. „Ein so eingeschränkter Speisezettel könne jedoch gerade bei Kindern schwierig sein“, räumte Hruz ein.

In der medikamentösen Behandlung werden vorwiegend die topischen Kortikosteroide Budosenid und Fluticason eingesetzt. Eine - kurzzeitige - systemische Therapie bleibt schweren Fällen vorbehalten. Bei Non-Respondern können auch Immunsuppressiva und Biologika erwogen werden. Bleibt die Behandlung erfolglos, sei dies nicht selten auf eine mangelnde Compliance zurückzuführen; eine entsprechende Beratung und Aufklärung sei daher unerlässlich, betonte Hruz. Bei Kindern mit schwerer Dysphagie werden in seltenen Fällen auch Dilatationen vorgenommen, die jedoch die Entzündung nicht beseitigen; daher sei eine Kombination mit einer antientzündlichen Medikation anzuraten, so Hruz.

Hruz P. Eosinophile Ösophagitis: Modelerkrankung unter den Eosinophilen Enteropathien
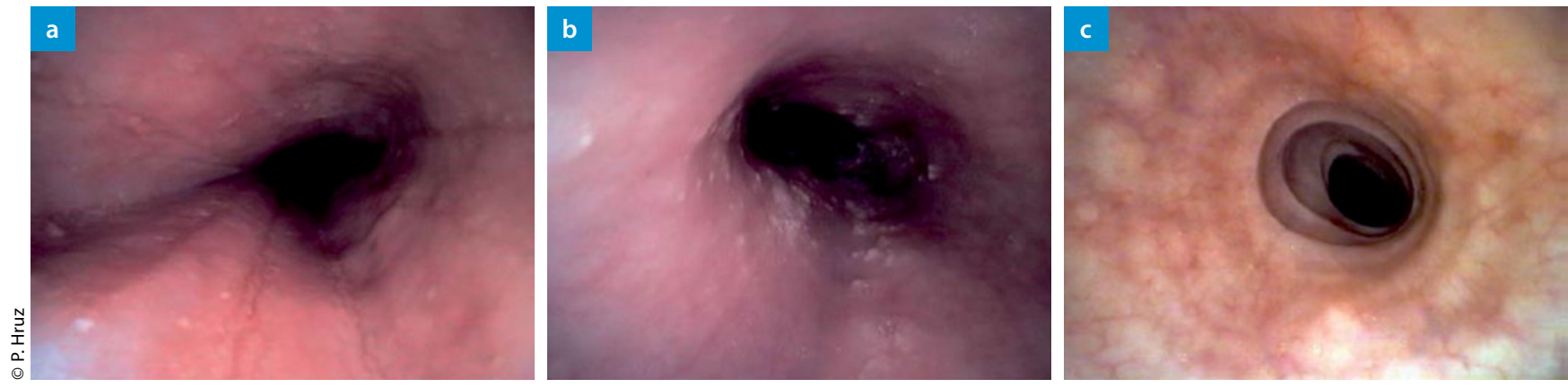

Endoskopische Zeichen einer eosinophilen Ösophagitis: Rötliche Längsfurchen (a), weißliche Exsudate (b), Trachealisierung (c)

\section{Buchtipp}

\section{Ein Draht zu Teenagern}

— Die verbale Kommunikation mit Jugendlichen ist nicht immer einfach. Vor allem im Alter von 13-16 Jahren neigen Jugendliche dazu, dem Gesprächspartner sehr direkt zu vermitteln, wenn sie sich nicht ernst genommen fühlen. Die Lektüre des Buches "Wie meinst Du das?" verhilft zur Selbstreflexion: Wie ist der eigene Gesprächsstil, entspricht er eher einem Monolog, wird dem Zuhören Raum gelassen, wird das Gegenüber respektiert? Das Buch gibt Anregungen, wie ein Gespräch aufzubauen ist und wie ein vertrauensvoller Rahmen geschaffen werden kann. Das wird sicher so manchen Jugendlichen aus seiner abwehrenden Haltung holen und einen positiven Dialog eröffnen.

Wie Erwachsene Teenager in der Kommunikation unterstützen können, ihr Gehirn einzuschalten, bzw. was sie vermeiden sollten, um den "off-Zustand" bei Jugendlichen zu verstärken, vermittelt Martine Delfos im ersten Teil des Buches ihren Lesern. Im zweite Teil erklärt die Autorin die Basis für einen Dialog: Respekt, Ernstnehmen des Gesprächpartners, dafür sorgen, dass das Gegenüber sich wohl fühlt, Interesse zeigen sowie die Fähigkeit zuzuhören.

Der letzte Abschnitt vermittelt praktische Anregungen: Gesprächstechniken inklusive
Fragetechnik und Körpersprache, die sokratische Methode, d.h. "entdecken lassen“, sowie eine Gesprächsführung nach Alter des Partners. Im Anhang findet der Leser Übungen in Kommunikation. Die Lektüre des Buches ist für alle Kollegen empfehlenswert, die im Umgang mit ihren jugendlichen Patienten Wert darauf legen, eine vertrauensvolle Basis aufzubauen. Dr. Kirsten Stollhoff

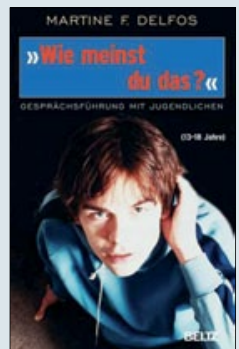

Martine F. Delfos

Wie meist Du das?

Gesprächsführung mit Jugendlichen

Beltz-Verlag, Weinheim

2012

$16,95 €$

ISBN 978-3-407-22181-0 